'Escuela de Enfermería, Pontificia Universidad Católica de Chile, Santiago, Chile. 2Departamento de Enfermedades Infecciosas del Adulto, Escuela de Medicina, Pontificia Universidad Católica de Chile.

${ }^{3}$ Unidad de Infectología. Complejo Asistencial Dr. Sótero del Río.

${ }^{4}$ CESFAM Juan Pablo II.

aPhD, Enfermera-matrona.

${ }^{b}$ Magíster, Enfermera-matrona. 'Alumna de Magíster, Enfermera. dMagíster, Enfermera. eMatrón(a)

*Proyecto financiado por el Fondo Nacional de Investigación y Desarrollo en Salud (FONIS) SA11/2137. La organización que proporcionó el financiamiento no tuvo influencia en el diseño de estudio; en la recolección, análisis o interpretación de los datos; en la preparación, revisión o aprobación del manuscrito.

Recibido el 30 de diciembre de 2013, aceptado el 29 de septiembre de 2014

Correspondencia a: Alejandra Araya. Vicuña Mackenna 4860, Macul, Santiago, Chile. Teléfono: (56)-2-2354-5834 Fax: (56)-2-2354-7025 aarayagu@uc.c

\section{Optimizando el acceso oportuno al test de ELISA para el diagnóstico del VIH: Recomendaciones desde los usuarios y profesionales de la Atención Primaria de Salud}

\author{
ALEJANDRA XIMENA ARAYA ${ }^{1, \mathrm{a}}$, MARIA-TERESA URRUTIA ${ }^{1, \mathrm{a}}$, \\ PAULA VEGA ${ }^{1, \mathrm{~b}}$, MIRIAM RUBIO ${ }^{1, \mathrm{c}}$, PAOLA CARRASCO ${ }^{1, \mathrm{~d}}$, \\ CARLOS PÉREZ C. ${ }^{2}$, ANA MARÍA FERNÁNDEZ ${ }^{3, \mathrm{e}}$, MARIANELA \\ OBREGÓN $^{2, \mathrm{e}}$, FRANCISCO ARANCIBIA ${ }^{4, \mathrm{e}}$, MARTIN LASSO B. ${ }^{3}$
}

Recommendations of health care users and professionals to achieve a timely access to HIV diagnosis

Background: Early HIV (human immunodeficiency virus) diagnosis optimizes therapies aimed at reducing viral load, increasing survival, lowering health costs and reducing the number of people infected with the virus. In Chile, despite widespread and readily available HIV testing, infected people continue to get tested in a late fashion and are usually diagnosed in advanced stages of the disease. Aim: To determine the elements that facilitate or impede a timely HIV testing and to evaluate how to improve the access to HIV testing. Material and Methods: Descriptive, in-depth interviews to 30 participants with unknown serology, 15 participants diagnosed at AIDS stage and 15 health care professionals working at a primary healthcare settings. Results: Users and professionals formulated three suggestions to improve timely access to ELISA test for HIV diagnosis. Namely, to inform users and professionals about the characteristics of the disease and diagnostic test, to offer fast and easy access to HIV testing, and to train the whole healthcare team about obtaining informed consent for testing. Conclusions: These recommendations should be implemented at healthcare centers to attain a timely HIV diagnosis.

(Rev Med Chile 2014; 142: 1284-1290)

Key words: Early diagnosis; HIV; Qualitative research.
E n Chile, a pesar de la disponibilidad del examen del test de ELISA para la detección del VIH, en la Atención Primaria de Salud (APS), el diagnóstico es aún tardío ${ }^{1,2}$. Según datos de la Cohorte Chilena de SIDA, 46\% de los usuarios que inician terapia antirretroviral han sido diagnosticados en etapas tardías ${ }^{3}$, hecho concordante con la realidad internacional ${ }^{4-6}$.

El diagnóstico tardío del VIH tiene implican- cias económicas para el país, aumentando los costos en salud ${ }^{7-9}$, por el contrario, el diagnóstico oportuno tiene como beneficio aumentar la tasa de sobrevida y disminuir la tasa de morbilidad asociada a la enfermedad ${ }^{7,8,10}$. El diagnóstico temprano de la enfermedad es crítico para prevenir la transmisión del $\mathrm{VIH}^{11,12}$. Las personas que no conocen su diagnóstico tienen un riesgo mayor de transmitir la enfermedad porque: a) están en la 
etapa de primoinfección o en etapas avanzadas de la enfermedad, con las consecuentes altas cargas virales $^{13,14}$; b) tienen más conductas sexuales de riesgo que aquellos que conocen su diagnóstico ${ }^{15,16}$; y c) tienen una prevalencia mayor de infecciones de transmisión sexual, lo cual aumenta la probabilidad de transmitir el $\mathrm{VIH}^{17}$.

El objetivo de este estudio es conocer los elementos facilitadores, dificultades y aquellas recomendaciones para mejorar el acceso oportuno al test del VIH desde la perspectiva de los usuarios como de los profesionales de la APS.

\section{Material y Método}

Estudio cualitativo, con entrevistas en profundidad, realizado en un total de 75 participantes del Servicio de Salud Metropolitano Sur-Oriente (SSMSO) en tres grupos de estudio: 1) 30 personas con serología desconocida; 2) 30 profesionales del área de salud pertenecientes a 2 Centros de Salud Familiar (CESFAM), y 3) 15 personas diagnosticadas en etapa de SIDA pertenecientes al Policlínico de Infectologia del Complejo Asistencial Dr. Sótero del Río. (CASR). Se entrevistaron a 15 participantes por cada tipo de centro para asegurar la saturación de los datos de este estudio basados en investigaciones anteriores en este mismo tipo de población.

Los criterios de inclusión para personas con serología desconocida fueron: (i) ser mayor de 18 años; (iii) tomarse el examen en los CESFAM, y (ii) no ser donante de sangre o estar embarazada. Los criterios de inclusión para personas viviendo con SIDA fueron: (i) ser mayor de 18 años; (ii) haber sido diagnosticado en etapa de SIDA según los criterios de clasificación de la $\mathrm{CDC}^{18}$; y (iii) para ambos usuarios, haberse tomado el test de manera espontánea, dado que el foco de este estudio está en aquellos usuarios que acuden espontáneamente a los CESFAM. Finalmente, los criterios de selección para los profesionales: (i) tener contrato de trabajo con una jornada mínima de $22 \mathrm{~h} /$ semanales, y (ii) poseer una antigüedad laboral mayor a un año en contacto directo con usuarios. La recolección de los datos fue realizada entre los meses de enero y septiembre de 2012. Se invitó a todos los usuarios que se tomaron el examen del VIH en los dos CESFAM y aquellos usuarios en control en el CASR. De las personas invitadas a participar, sólo cinco rechazaron ser entrevistadas, aludiendo a problemas de tiempo para realizar la entrevista. Para la entrevista en profundidad, se utilizó una guía semi-estructurada con cinco preguntas conductoras relacionadas a los facilitadores, dificultades y recomendaciones para mejorar el acceso oportuno al test del VIH en APS (Tabla 1). Las entrevistas duraron en promedio 40 min y fueron grabadas y transcritas.

Para el análisis de los datos se utilizó la técnica de análisis de contenido de Krippendorff ${ }^{19}$. Cada entrevista fue leída por cinco investigadores por separado. La primera lectura fue sin interpretación; luego se realizó una segunda lectura identificando las principales dimensiones; y la tercera lectura para corroborar las dimensiones y categorías. Luego cada investigador propuso las dimensiones/ categorías encontradas llegando a consenso en reunión de equipo. Los resultados obtenidos fueron corroborados por los 19/30 usuarios de la APS, 6/15 del CASR, y 16/30 profesionales.

\section{Tabla 1. Guía de entrevista en profundidad de los usuarios y profesionales}

Elementos facilitadores del acceso al examen del VIH

Elementos que dificultan el acceso al examen del VIH

Sugerencias para mejorar el acceso oportuno al examen del VIH
Según su experiencia, ¿cuál o cuáles serían los elementos personales o del centro de salud que le ayudaron a tomarse este test?

En su opinión, ¿Qué otros elementos son importantes de ser incorporados para facilitar el acceso a la toma de este test en este centro de salud?

Según su experiencia, ¿cuál o cuáles serían las dificultades que usted ha tenido para tomarse este test en este centro de salud?

¿Cómo cree usted que estas dificultades pueden ser solucionadas para facilitar el acceso al test en este centro de salud?

Señale cuál o cuáles son las sugerencias que usted haría para mejorar el acceso oportuno al test de ELISA para el diagnóstico del VIH en el centro de salud que usted se atiende 
Una vez analizadas las entrevistas en cada grupo, por separado, se procedió al proceso de triangulación de la información, seleccionando aquellos temas presentes en los tres grupos de estudio y que confluyeran en las mismas dimensiones. Cada investigador propuso los temas/dimensiones lográndose el consenso unánime en ello.

Este estudio contó con la aprobación del Comité de Evaluación Ético-Científico del SSMSO y de la Escuela de Enfermería de la Pontificia Universidad Católica de Chile.

\section{Resultados}

La caracterización de los usuarios con serología desconocida y de aquellas personas viviendo con SIDA se muestra en conjunto dada la homogeneidad de los resultados. La mediana de edad de los usuarios fue 38 años, con un rango entre 19 y 77 años. Del total de usuarios 25 eran hombres y el resto mujeres; 13/45 eran hombres que tienen sexo con hombres, 30/45 heterosexuales y el resto bisexuales. En relación a la ocupación, 33/45 reportaron algún tipo de trabajo remunerado, 5/45 estaban cesantes, 2/45 jubilados y el resto no respondió. En relación a conductas de riesgo para contagio VIH, ninguno de ellos reportó ser usuarios de drogas endovenosas y 26/45 no usaron preservativos durante el último año. En relación a los profesionales, la mediana de edad fue de 32 años, con un rango entre 25 y 57 años. Del total $22 / 30$ eran mujeres y el resto hombres. La media- na de años trabajando en contacto directo con adultos en el nivel primario de atención fue de 6 años, con un rango entre 2 y 27 años. Del total de profesionales, 7 eran enfermeros(as), 6 médicos, 6 asistentes sociales, 5 psicólogos, 3 matrones(as) y el resto kinesiólogos(as).

La Tabla 2 presenta los facilitadores, dificultades y recomendaciones para el acceso oportuno al examen del VIH en la APS. Dentro de los elementos que facilitaban su toma oportuna se destaca la cercanía del equipo de salud y la confidencialidad del examen. Los usuarios reconocieron que la orientación, calidez y acogida del profesional en la consejería, junto con el ofrecimiento que los profesionales hacen del examen al momento del control de salud actúan como elementos facilitadores para tomárselo. Que el examen pueda ser tomado en el CESFAM es otro factor que facilita el acceso a éste. Los profesionales reconocieron que el vínculo que se establece con los usuarios y la conectividad de los profesionales al interior del CESFAM como elementos facilitadores.

Dentro de las dificultades encontradas para el acceso oportuno al examen del VIH se encuentran el miedo a la discriminación, falta de información, la burocracia del proceso, la falta de tiempo y el miedo al diagnóstico y/o a vivir con VIH. En relación al miedo a la discriminación, esto se relaciona a que ambos grupos reconocen que el hecho de solicitar el examen en los CESFAM les da desconfianza y miedo por temor a ser reconocidos por sus vecinos.

Tanto los profesionales como los usuarios

Tabla 2. Dimensiones y categorías sobre los facilitadores, dificultades y recomendaciones desde la perspectiva de los usuarios y profesionales $(n=75)$

\begin{tabular}{ll}
\hline Dimensión & Categoría \\
$\begin{array}{l}\text { Facilitadores para tomarse el } \\
\text { examen del VIH }\end{array}$ & $\begin{array}{l}\text { Cercanía del equipo de salud } \\
\text { Confidencialidad del examen } \\
\text { Realización examen en el CESFAM }\end{array}$ \\
$\begin{array}{l}\text { Dificultades para tomarse el } \\
\text { examen del VIH }\end{array}$ & $\begin{array}{l}\text { Miedo discriminación de los vecinos } \\
\text { Falta de información sobre el test } \\
\text { Burocracia para tomarse el test } \\
\text { Falta de tiempo (usuarios/profesionales) }\end{array}$ \\
& Miedo al diagnóstico (vivir con VIH) \\
Recomendaciones para mejorar & Informar a los usuarios sobre el examen del VIH y educarlos sobre la enfermedad \\
el acceso al examen de VIH & Aumentar la cobertura del examen del VIH, ofreciéndolo de manera expedita y rápida \\
& Capacitación al equipo de salud en la toma del examen del VIH
\end{tabular}


fueron consistentes en identificar que la falta de información, la burocracia en el proceso de toma del test y la falta de tiempo como barreras para acceder oportunamente al test del VIH. La falta de información alude al desconocimiento de los usuarios sobre dónde, cuándo y quién se puede tomar el examen del VIH, junto con la falta de educación sobre las vías de infección, protección y su tratamiento. Por ejemplo, tanto profesionales como usuarios desconocían que este examen puede tomarse en sus respectivos CESFAM.

En relación a la burocracia, los usuarios identificaron aspectos administrativos tediosos y dificultades en la navegación por los CESFAM como aspectos obstaculizadores. Por ejemplo, los usuarios señalaron que no pueden acceder a tomarse el examen cualquier día o cualquier horario. Esta situación también fue reconocida por los profesionales de la salud, quienes señalaron que los CESFAM no tienen la disponibilidad instalada de tomar el examen en modalidad libre demanda, ya que dependen de la programación de horas de consentimiento informado y del servicio de transporte de las muestras de sangres que son derivadas al laboratorio del SSMSO.

Con relación a la falta de tiempo, los usuarios reconocen como barreras las instancias de solicitud de hora, consentimiento informado y tomarse el examen como tres instancias que necesitan de su tiempo, muchas veces, en dos días distintos. Por su lado, los profesionales, reconocieron que no tienen el tiempo disponible para acceder a solicitudes de este examen todos los días en cualquier momento, dado el tiempo que requiere la firma del consentimiento informado.

Finalmente, el miedo al diagnóstico es una dificultad identificada sólo por los usuarios de la APS y del CASR, no así por los profesionales. Se refiere al temor asociado a que el resultado del examen sea positivo y, consecuentemente, al diagnóstico de ser portador del VIH.

Son tres las recomendaciones para mejorar el acceso oportuno al examen del VIH: informar a los usuarios sobre el examen y educarlos sobre la enfermedad; aumentar la cobertura del examen, ofreciéndolo de manera expedita y rápida; y capacitación al equipo de salud en la toma del mismo. En relación a la categoría de informar a los usuarios sobre el examen del VIH y educarlos sobre la enfermedad, los usuarios refirieron necesitar una información sobre el procedimiento de tomarse el examen en los CESFAM y educación continua sobre la enfermedad del VIH, su tratamiento y expectativa de vida. Para cumplir con estos fines, los usuarios reconocieron la importancia de usar medios de difusión al interior del CESFAM, además de la consejería de los profesionales que los atienden.

Tanto profesionales como usuarios coincidieron que es necesario aumentar la cobertura del examen del VIH, ofreciéndolo de manera expedita y rápida. Por un lado, los usuarios sugirieron que la solicitud de la hora, el consentimiento informado y la toma del examen sea un procedimiento que se realice en un mismo día. En este sentido, los profesionales identificaron que es necesario contar con más horas para realizar el consentimiento informado y que esta solicitud sea realizada por los profesionales que la pesquisan, evitando la derivación al interior de los CESFAM. Por otro lado, los profesionales propusieron aumentar la cobertura a través dela inclusión de la oferta del test en los Exámenes de Medicina Preventiva del Adulto. De esta forma el examen se "normalizaría" $y$, por ende, se transformaría en un examen más conocido, habitual y menos temido.

La tercera categoría que emerge como recomendación es la capacitación al equipo de salud en la toma del examen. Por ejemplo, dónde se toma, quién lo puede solicitar, horarios de atención para que los usuarios no se pierdan al interior del CESFAM al momento de ser derivados. En este mismo sentido, es necesario que todos los profesionales que por normativa ministerial puedan realizar el consentimiento informado, estén capacitados para esto. También señalan la importancia de tener espacios de conversación al interior del equipo sobre las formas de sugerir el examen a sus usuarios.

\section{Discusión}

La epidemia del VIH/SIDA puede ser substancialmente disminuida si se aumenta el número de personas que conocen su diagnóstico ${ }^{20}$, de hecho la tasa de transmisión es 3,5 veces mayor en el grupo de personas que no conocen su diagnóstico de $\mathrm{VIH}^{20}$, por lo que se hace necesario expandir la cobertura del examen. Para aumentar el acceso oportuno al test del VIH, informar a los usuarios sobre el examen y educarlos sobre la enfermedad; aumentar la cobertura del examen, ofreciéndolo 
de manera expedita y rápida; y capacitar al equipo de salud en la toma del mismo, son tres recomendaciones entregadas por los tres grupos de estudio.

La primera recomendación es informar a los usuarios sobre el examen y educarlos sobre la enfermedad. El desconocimiento de los usuarios sobre la disponibilidad del test ha sido descrita como una de las principales barreras de acceso al examen $^{21,22}$. Otro aspecto importante es entregar información a los usuarios sobre la enfermedad, sus factores de riesgo, tratamiento, expectativas de vida y sobre los servicios existentes para aquellas personas con $\mathrm{VIH}^{23}$. Mientras los usuarios estén informados de esos aspectos se sentirán más confiados de tomarse el examen del VIH ${ }^{24,25}$, disminuyendo las barreras de miedo discriminación, falta de información sobre el test y miedo al diagnóstico/vivir con VIH.

Una segunda recomendación es aumentar la cobertura del examen, ofreciéndolo de manera expedita y rápida, disminuyendo los procesos administrativos que obstaculizan su acceso oportuno. La solicitud del examen a través del "mesón" de los CESFAM hace que los usuarios se alejen de solicitarlo, para evitar ser vistos, lo que les genera vergüenza y miedo. Implementar una solicitud vía telefónica puede ser una estrategia que disminuya esta barrera de acceso. En este sentido, que el examen pueda ser tomado a cualquier hora del día es un aspecto crucial de abordar en los CESFAM. Los profesionales destacan que el envío de la muestra a laboratorio es uno de los elementos que dificultan que este examen pueda ser tomado en modalidad libre demanda. En relación al envío de la muestra, es necesario que los CESFAM sean actualizados sobre la preservación de la muestra y puedan disponer de medios de transporte que permitan distribuir oportunamente las muestras de sangre, disminuyendo barreras de acceso relacionados con la burocracia y la falta de tiempo para el acceso oportuno a este examen.

La tercera recomendación alude a la capacitación al equipo de salud en la toma del examen. Que el profesional que ofrezca el examen sea el que realice el consentimiento informado, orientado a disminuir la burocracia y la falta de tiempo identificada como barreras de acceso oportuno al examen del VIH. Considerar la capacitación en el proceso de consentimiento informado por todos aquellos profesionales que por decreto ministerial están habilitados para hacerlo, de esta forma contribuir a aumentar las horas disponibles y, por ende, aumentar el acceso a este test. Otro aspecto es informar a los profesionales sobre la disponibilidad del examen en sus respectivos centros es una estrategia que ha demostrado aumentar la cobertura de este y su diagnóstico oportuno ${ }^{26}$. Algunas personas que han sido diagnosticadas tardíamente han sido evaluados por profesionales de la salud un año anterior a su diagnóstico ${ }^{27,28}$, evidenciado el retraso de la oportunidad de haber sido diagnosticados oportunamente ${ }^{29}$. Junto con esto, los profesionales identifican la falta de actualización sobre los grupos de riesgo y de conocimiento sobre el examen como elementos que dificultan ofrecerlo a los usuarios que atienden ${ }^{30}$.

Finalmente, en relación a los aspectos facilitadores para tomarse el examen, la cercanía del equipo de salud, la confidencialidad del examen y la realización examen en el CESFAM son tres aspectos fundamentales de mantener. Informar que el proceso de toma del test es privado y confidencial, es clave para que las personas decidan acceder al examen del $\mathrm{VIH}^{31-33}$, ya que permite darles confianza y disminuir el miedo a ser descubiertos por otros.

Cabe destacar que en todas las categorías hubo un acuerdo entre los tres grupos de estudio, sin embargo, el miedo a vivir con VIH fue sólo identificado por los usuarios tanto de serología desconocida como de aquellos viviendo con VIH. Esta diferencia con el grupo de profesionales se puede explicar, dado que los profesionales relacionan al VIH como una enfermedad crónica, con la cual se es posible vivir en buenas condiciones de salud. A diferencia de los usuarios, que por lo general relacionan a la enfermedad como una sentencia de muerte al momento del diagnóstico.

Dada la metodología utilizada en esta investigación los resultados no son aplicables a la realidad nacional, sin embargo, es el primer trabajo en Chile que permite analizar la perspectiva tanto de usuarios como de profesionales en relación a esta temática.

Hacer el examen del VIH más accesible a la comunidad es una tarea pendiente y una prioridad para la salud pública en Chile. Informar a usuarios y profesionales sobre el examen y la enfermedad, contar con una oferta del examen del VIH rápida y expedita y capacitar al equipo de salud en la toma del examen del VIH son las tres recomendaciones necesarias de implementar con el objetivo de au- 
mentar el diagnóstico de personas con VIH y, por lo tanto, poder ofrecerles un tratamiento oportuno que mejore sus expectativas de sobrevida.

\section{Referencias}

1. Ley 19.779 y Reglamento del Examen para la Detección del Virus de Inmunodeficiencia humana, Decreto No 182 de 2005.

2. MINSAL. Guía clínica: Síndrome de inmunodeficiencia adquirida VIH/SIDA. Revista Chilena de Infectología 2010; 27 (3): 239-76.

3. Wolff MJ, Cortés CP, Shepherd BE, Beltrán CJ. Longterm outcomes of a national expanded access program to antiretroviral therapy: the Chilean AIDS cohort. J Acquir Immune Defic Syndr 2010; 55 (3): 368-74.

4. Ndiaye B, Salleron J, Vincent A, Bataille P, Bonnevie F, Choisy $\mathrm{P}$, et al. Factors associated with presentation to care with advanced HIV disease in Brussels and Northern France: 1997-2007. BMC Infect Dis 2011; 11: 11.

5. Yazdanpanah Y, Lange J, Gerstoft J, Cairns G. Earlier testing for HIV-how do we prevent late presentation? Antivir Ther 2010; 15 Suppl 1: 17-24.

6. Mayben JK, Kramer JR, Kallen MA, Franzini L, Lairson DR, Giordano TP. Predictors of delayed HIV diagnosis in a recently diagnosed cohort. AIDS Patient Care STDS 2007; 21 (3): 195-204.

7. Chadborn TR, Delpech VC, Sabin CA, Sinka K, Evans BG. The late diagnosis and consequent short-term mortality of HIV-infected heterosexuals (England and Wales, 2000-2004). AIDS 2006; 20 (18): 2371-9.

8. Krentz HB, Auld MC, Gill MJ. The high cost of medical care for patients who present late (CD4 $<200$ cells/microL) with HIV infection. HIV Med 2004; 5 (2): 93-8.

9. Donnell D, Baeten JM, Kiarie J, Thomas KK, Stevens W, Cohen CR, et al. Heterosexual HIV-1 transmission after initiation of antiretroviral therapy: a prospective cohort analysis. Lancet; 375 (9731): 2092-8.

10. Castilla J, Sobrino P, De La Fuente L, Noguer I, Guerra L, Parras F. Late diagnosis of HIV infection in the era of highly active antiretroviral therapy: consequences for AIDS incidence. AIDS 2002; 16 (14): 1945-51.

11. Glasman LR, Weinhardt LS, Difranceisco W, Hackl KL. Intentions to seek and accept an HIV test among men of Mexican descent in the Midwestern USA. AIDS Care 2010; 22 (6): 718-28.

12. Adler A, Mounier-Jack S, Coker RJ. Late diagnosis of HIV in Europe: definitional and public health challenges. AIDS Care 2009; 21 (3): 284-93.

13. Wawer MJ, Gray RH, Sewankambo NK, Serwadda D, Li
$\mathrm{X}$, Laeyendecker O, et al. Rates of HIV-1 transmission per coital act, by stage of HIV-1 infection, in Rakai, Uganda. J Infect Dis 2005; 191 (9): 1403-9.

14. Brenner BG, Roger M, Routy JP, Moisi D, Ntemgwa M, Matte C, et al. High rates of forward transmission events after acute/early HIV-1 infection. J Infect Dis 2007; 195 (7): 951-9.

15. Marks G, Crepaz N, Senterfitt JW, Janssen RS. Metaanalysis of high-risk sexual behavior in persons aware and unaware they are infected with HIV in the United States: implications for HIV prevention programs. J Acquir Immune Defic Syndr 2005; 39 (4): 446-53.

16. Schwarcz S, Richards TA, Frank H, Wenzel C, Chin Hsu L, Chin CS, et al. Identifying barriers to HIV testing: personal and contextual factors associated with late HIV testing. AIDS Care 2011; 18: 1-9.

17. Corey L, Wald A, Celum CL, Quinn TC. The effects of herpes simplex virus- 2 on HIV-1 acquisition and transmission: a review of two overlapping epidemics. J Acquir Immune Defic Syndr 2004; 35 (5): 435-45.

18. CDC. 1993 revised classification system for HIV infection and expanded surveillance case definition for AIDS among adolescents and adults. MMWR Recomm Rep 1992; 18 (4): 1-19.

19. Krippendorff K. Content analysis: An introduction to its methodology. Thousands Oaks: Sage Publications; 2004.

20. Marks G, Crepaz N, Janssen RS. Estimating sexual transmission of HIV from persons aware and unaware that they are infected with the virus in the USA. AIDS. 2006; 20 (10): 1447-50.

21. Trieu SL. Partner communication and factors associated with the decision to obtain an HIV Test among Chinese/ Chinese American community college students in Northern California. Dissertation Abstracts International: Section B: The Sciences and Engineering. [Dissertation]. 2008; 69 (4-B): 2279.

22. Mitra D, Jacobsen M, O'Connor A, Pottie K, Tugwell P. Assessment of the decision support needs of women from HIV endemic countries regarding voluntary HIV testing in Canada. Patient Education and Counseling 2006; 63 (3): 292-300.

23. Daftary A, Padayatchi N, Padilla M. HIV testing and disclosure: A qualitative analysis of TB patients in South Africa. AIDS Care-Psychological and Socio-Medical Aspects of AIDS/HIV. 2007; 19 (4): 572-7.

24. Aho J, Nguyen VK, Diakite S, Sow A, Koushik A, Rashed S. High acceptability of HIV voluntary counselling and testing among female sex workers: impact of individual and social factors. HIV Med 2012; 13 (3): 156-65.

25. Nyanzi-Wakholi B, Lara AM, Watera C, Munderi P, Gilks C, Grosskurth H. The role of HIV testing, counselling, 
and treatment in coping with HIV/AIDS in Uganda: a qualitative analysis. AIDS Care 2009; 21 (7): 903-8.

26. Njau B, Watt M, Ostermann J, Manongi R, Sikkema K. Perceived acceptability of home-based couples voluntary HIV counseling and testing in northern Tanzania. AIDS Care. 2012; 24 (4): 413-9.

27. Sudarshi D, Pao D, Murphy G, Parry J, Dean G, Fisher M. Missed opportunities for diagnosing primary HIV infection. Sex Transm Infect 2008; 84 (1): 14-6.

28. Sullivan AK, Curtis H, Sabin CA, Johnson MA. Newly diagnosed HIV infections: review in UK and Ireland. BMJ 2005; 330 (7503): 1301-2.

29. Kuo AM, Haukoos JS, Witt MD, Babaie ML, Lewis RJ. Recognition of undiagnosed HIV infection: an evaluation of missed opportunities in a predominantly urban minority population. AIDS Patient Care STDS 2005; 19 (4): 239-46.
30. Burke RC, Sepkowitz KA, Bernstein KT, Karpati AM, Myers JE, Tsoi BW, et al. Why don't physicians test for HIV? A review of the US literature. AIDS 2007; 21 (12): 1617-24.

31. Ransom JE, Siler B, Peters RM, Maurer MJ. Worry: Women's experience of HIV testing. Qualitative Health Research 2005; 15 (3): 382-93.

32. Barnabas Njozing N, Edin KE, Hurtig AK. 'When I get better I will do the test': Facilitators and barriers to HIV testing in Northwest Region of Cameroon with implications for TB and HIV/AIDS control programmes. SAHARA J: journal of Social Aspects of HIV/AIDS Research Alliance/SAHARA, Human Sciences Research Council 2010; 7 (4): 24-32.

33. Castle S. Doubting the existence of AIDS: A barrier to voluntary HIV testing and counselling in urban Mali. Health Policy and Planning 2003; 18 (2): 146-55. 